\title{
La reforma de la Administración pública en Rumanía
}

Marius Profiroiu y Tudorel Andrei*

Palabras clave: Reforma administrativa, gestión pública, poderes locales, modernización, democratización, integración europea, lucha contra la corrupción

\section{Marco de la Reforma de la Administración Pública (RAP)}

En Diciembre de 2004 Rumanía concluyó las negociaciones para la adhesión a la Unión Europea, pero aún hay muchas cosas por hacer antes de que se cumpla la actual fecha de acceso, que será, probablemente, enero de 2007. La integración en las estructuras europeas requiere el desarrollo de una Administración pública con valores coincidentes a los del Espacio Administrativo Europeo y también capaz de permitir a Rumanía cumplir los requisitos de una pertenencia completa a la UE.

La reforma de la Administración pública refleja cambios sustantivos en sus principales componentes, tanto a nivel administrativo local como del gobierno central, y en la prestación de servicios públicos en general. Por otro lado, la consolidación democrática exige el desarrollo de una nueva relación entre los ciudadanos y la Administración, el refuerzo del papel de las autoridades y la redefinición de los lazos con la sociedad civil y los cargos públicos electos.

\subsection{La capacidad administrativa y la Reforma de la Administración Pública}

En los debates sobre la Reforma de la Administración Pública en Rumanía a menudo se ha producido alguna confusión sobre lo que en realidad ésta implica. En términos concretos significa algo más que el tipo de reformas requeridas para mejorar la capacidad administrativa. Hay dos categorías diferentes de ideas sobre la organización del sector público, aunque están conectadas funcionalmente.

* Profesores de la Facultad de Management., Academia de Estudios Económicos de Bucarest. Marius Profiroin ba sido Secretario de Estado de Administraciones Públicas del Gobierno rumano durante la legislatura 2001-2005. 
RAP es un concepto amplio; se refiere a todos los aspectos de la organización del sector público incluyendo el conjunto de la arquitectura de los ministerios y las agencias, las organizaciones, sistemas, estructuras, procesos, incentivos, así como los acuerdos para mantener la gobernabilidad y los que permiten la reforma ocasional del sistema. De un lado, el término Administración se refiere al modo en que la coordinación de las acciones del sector público está formalmente regulada, ordenada y organizada. De otro, la capacidad administrativa es una valoración del funcionamiento de la jerarquía de cargos del sector público, pero esto es, desde luego, sólo un elemento del amplio abanico incluido en la RAP.

No obstante, la capacidad administrativa es crucial para la reforma y para el funcionamiento del Estado, pero, como se ha apuntado, sólo es una parte de una visión más amplia. Por sí misma no será eficaz para dar los resultados que se esperan de una Administración moderna. De hecho, un incremento de la capacidad administrativa, por sí mismo, puede ser tanto un estorbo como una ayuda para alcanzar resultados. Depende, en parte, de cómo se organice y dirija, y también de cómo se dote de personal y la actitud de este personal para llevar a cabo sus funciones.

Para apoyar el cambio fundamental del sistema administrativo, de acuerdo con los requisitos del proceso de reforma, un conjunto consistente de medidas ha de implementarse en un marco temporal preciso, en las áreas de la reforma del sector público -destinado a crear un cuerpo de servidores públicos profesional, estable y políticamente neutral-, la Administración pública local -destinado a continuar el proceso de descentralización y desconcentración de los servicios públicos -y la reforma del gobierno central- destinada a mejorar el proceso de formulación de políticas.

Un proceso coherente y creíble de reforma de la Administración pública que compromete tanto al sistema administrativo como político requiere el desarrollo de una red estable que promueva el cambio, que implique a los principales valedores en este proceso.

En la práctica, la red de reformadores puede ponerse en marcha simplemente tratando de desarrollar las capacidades de gestión y resolviendo el problema de cómo instalar estas nuevas capacidades dentro de las jerarquías del Estado ya existentes. Este proceso sacará a la luz el tipo de problemas que otros países han tenido que resolver y reducirá el ciclo de aprendizaje que ha de atravesar Rumanía. En 2002 una red nacional de modernizadores que cubría tanto la Administración local como la central fue establecida con el apoyo de expertos subvencionados por la UE. La red consta de más de 380 funcionarios públicos que trabajan en ministerios, provincias y comunidades locales.

\subsection{Análisis estadístico de las características del proceso de implementación de la RAP}

\section{Características generales}

En 2005, el Instituto Europeo de Rumanía financió una investigación para evaluar el proceso de implementación de las medidas de la RAP y el desarrollo de la capacidad de la red de modernizadores. El estudio también incluyó datos recogidos de dos muestras representativas de alcaldes de municipios y modernizadores de los ministerios y las provincias. 
Las dos primeras muestras fueron identificadas por una técnica de muestreo de dos niveles y representó el $9 \%$ del total de la población estudiada. El error estimado a nivel de las poblaciones de referencia es de 1.2\% para la primera muestra y de $1.8 \%$ para la segunda.

En la elaboración de los cuestionarios se consideraron los dos siguientes aspectos:

- La medición de la opinión de importantes valedores del proceso sobre asuntos de la actual RAP: gestión del sector público, formación de los funcionarios públicos, RAP local y proceso de descentralización, formación de los funcionarios locales electos sobre asuntos específicos de desarrollo local, corrupción, cambios en el personal técnico bajo presiones políticas, comunicación y coordinación del proceso de reforma, etc.

- Medición de la convergencia de la visión sobre los aspectos de la reforma entre los funcionarios públicos, incluyendo la red de modernizadores, y los políticos y los alcaldes.

\section{Percepción del proceso de RAP}

Tanto el personal técnico como los funcionarios electos perciben la reforma de la administración como un proceso que no ha traído los cambios esperados. Así, la mitad de los alcaldes tiene una opinión negativa sobre los cambios en la Administración pública mientras que un 36,4\% de los modernizadores cree que la Administración pública está cambiando en pequeña medida. Más aún, entre los dos principales actores del proceso de reforma local, los modernizadores de las prefecturas y los consejos provinciales y los alcaldes de las comunidades locales, hay diferencias significativas de percepción.

TABLA 1

¿Cree que la administración pública está llevando a cabo un proceso de reforma amplio?

\begin{tabular}{|c|c|c|c|c|c|}
\hline \multirow[b]{2}{*}{ Opciones de requesta } & \multicolumn{2}{|c|}{ Alcaldes } & \multicolumn{2}{|c|}{ Modernizadores } & \multirow[b]{2}{*}{$\begin{array}{c}\text { Balan } \\
\text { ce } \\
(\%)\end{array}$} \\
\hline & $\begin{array}{c}\text { Frecuencia } \\
\text { relativa } \\
(\%)\end{array}$ & $\begin{array}{c}\text { Frecuencia } \\
\text { relativa } \\
\text { acumulada } \\
(\%)\end{array}$ & $\begin{array}{c}\text { Frecuencia } \\
\text { relativa } \\
(\%)\end{array}$ & $\begin{array}{c}\text { Frecuencia } \\
\text { relativa } \\
\text { acumulada } \\
(\%)\end{array}$ & \\
\hline 1 & 2 & 3 & 4 & 5 & $6=4-2$ \\
\hline No & 3.6 & 3.6 & 0.0 & 0.0 & 3.6 \\
\hline En pequeña medida & 46.6 & 50.2 & 36.4 & 36.4 & 10.2 \\
\hline En gran medida & 41.9 & 92.1 & 54.5 & 90.9 & -12.6 \\
\hline Cambios radicales & 7.1 & 99.2 & 9.1 & 100.0 & -2.0 \\
\hline $\mathrm{NS} / \mathrm{NC}$ & 0.8 & 100.0 & 0.0 & 100.0 & 0.8 \\
\hline Total & 100.0 & - & 100.0 & - & - \\
\hline
\end{tabular}




\section{GRÁFICO 1}

¿Cree que la Administración pública está llevando a cabo un proceso de reforma amplio?

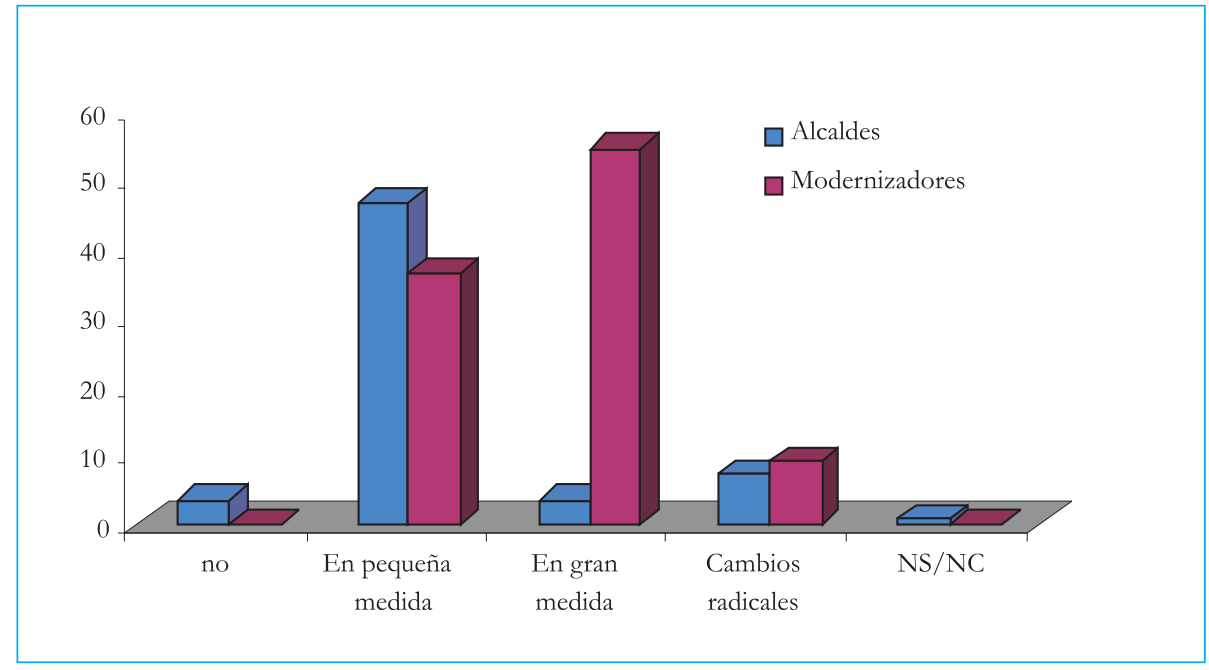

Varias son las posibles explicaciones de esta situación:

- Falta de comunicación entre los grupos de modernizadores locales y los alcaldes de comunidades locales sobre asuntos específicos de la reforma.

- Alguna de las reformas emprendidas no tienen todavía efectos significativos a nivel local: el proceso de reforma es a largo plazo.

- El mensaje que viene del gobierno central no está acompañado por una campaña continuada de información y sesiones de formación destinadas a los funcionarios locales electos sobre componentes específicos de la reforma.

En cualquier caso, la falta de una campaña de información sobre las medidas de reforma que han de tomarse en el próximo período y que afectan a la Administración local, proporciona una explicación para el vacío existente entre las expectativas y los cambios percibidos por los alcaldes.

TABLA 2

¿En qué medida los cambios colman sus expectativas?

\begin{tabular}{|l|c|c|}
\hline \multicolumn{1}{|c|}{ Opciones de respuesta } & Frecuencia relativa (\%) & $\begin{array}{c}\text { Frecuencia relativa } \\
\text { acumulada (\%) }\end{array}$ \\
\hline En absoluto & 5.5 & 5.5 \\
\hline En pequeña medida & 48.6 & 54.2 \\
\hline En gran medida & 39.1 & 93.3 \\
\hline Completamente & 6.3 & 99.6 \\
\hline NS/NC & 0.4 & 100.0 \\
\hline Total & 100.0 & - \\
\hline
\end{tabular}




\section{Introducción de berramientas modernas de gestión}

Para apoyar el proceso de RAP, la Unidad Central para la Reforma de la Administración Pública (UCRAP) inició la introducción de dos herramientas innovadoras tanto para el gobierno central como las prefecturas y concejos provinciales: los Programas Plurianuales de Modernización (PPM) y el Marco Común de Evaluación (MCE).

- Los Programas Plurianuales de Modernización (prolongados durante tres años), se ejecutan a través de tres instrumentos:

- La Estrategia de Modernización para cada institución, que describe el entorno interno, las prioridades sectoriales identificadas, las acciones de modernización y los procesos de implementación, control y evaluación.

- El Plan de Acción, que incluye las medidas pronosticadas, los resultados esperados, los indicadores de progreso, fechas límite, responsabilidad y fuentes de financiación.

- El Informe Anual de Control, que establece en qué medida los objetivos esperados se han cumplido, reelaborando el calendario de las acciones pendientes e introduciendo nuevas prioridades en el plan de acción para el siguiente año.

La implantación de los PPM en ministerios, prefecturas y provincias, comenzó en Junio, cuando se les solicitó un borrador de estrategias para acelerar la RAP en las políticas de su competencia.

El cuestionario distribuido a la red de modernizadores incluía dos preguntas sobre su opinión acerca de la implementación y el conjunto de resultados de esta herramienta. Las conclusiones más importantes se presentan a continuación:

- La opinión general es favorable a la implementación de los PPM en los organismos públicos. Es notable que ninguno de los entrevistados considerara la implementación de los PPM como «sólo una actividad burocrática).

- Cerca de la mitad de los modernizadores piensa que es una herramienta efectiva en el proceso de reforma de la Administración pública.

- La otra mitad es también favorable a la implementación de PPM, pero señala dificultades significativas en el actual estado de la RAP. Esto se relaciona con la falta de un mecanismo de financiación específicamente creado para apoyar las medidas de modernización establecidas en el Plan de Acción de los PPM.

- Los PPM han producido resultados positivos en el área de la comunicación e IT.

- Resultados no tan positivos se alcanzaron en el área de la gestión de recursos humanos. Así, más de la mitad de los sujetos entrevistados consideró que los resultados fueron insignificantes a partir de la implementación de los PPM.

\section{GRÁFICO 2}

¿Qué describe mejor la implementación de los PPM en su organismo?

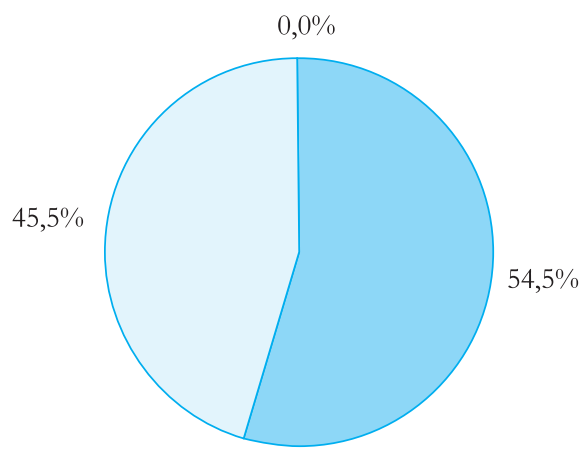

una actividad burocrática

$\square$ una herramienta de modernización difícil de implementar en las actuales circunstancias

$\square$ una herramienta efectiva en el proceso de modernización 
TABLA 3

¿En qué medida el PPM contribuye a mejorar lo signiente?

\begin{tabular}{|l|c|c|c|c|}
\hline & $\begin{array}{c}\text { Organización } \\
\text { interna } \\
\mathbf{( \% )}\end{array}$ & $\begin{array}{c}\text { Gestión de } \\
\text { Recursos } \\
\text { Humanos } \\
(\%)\end{array}$ & $\begin{array}{c}\text { Herramientas } \\
\text { de gestión } \\
\text { pública (\%) }\end{array}$ & $\begin{array}{c}\text { Comunicación } \\
\text { y TIC } \\
\mathbf{( \% )}\end{array}$ \\
\hline En absoluto & 0.0 & 4.5 & 0.0 & 4.5 \\
\hline En grado insignificante & 9.1 & 13.6 & 13.6 & 0.0 \\
\hline Moderadamente & 27.3 & 36.4 & 36.4 & 36.4 \\
\hline Bastante & 27.3 & 22.7 & 36.4 & 22.7 \\
\hline En gran medida & 27.3 & 13.6 & 4.5 & 31.8 \\
\hline NS/NC & 9.1 & 9.1 & 9.1 & 4.5 \\
\hline Total & 100.0 & 100.0 & 100.0 & 100.0 \\
\hline
\end{tabular}

El Marco Común de Evaluación (MCE) mide el funcionamiento de la organización, haciendo uso de una técnica de análisis diagnóstico. Esta herramienta, diseñada en el 2000 por el Grupo de Servicios Públicos Innovadores (Innovative Public Services Group) de la Comisión Europea, tiene cuatro líneas de trabajo principales:

- Aspectos específicos de las organizaciones del sector público;

- Mejora del funcionamiento de la organización;

- Coordinación de varios modelos de la gestión de la calidad;

- Examen de los indicadores de referencia (benchmarking) de las organizaciones del sector público.

La segunda versión del MCE está siendo usada por la Administración central y local, pero también por compañías privadas en la Unión Europea.

Durante el 2004, esta herramienta se introdujo como proyecto piloto de base en nueve divisiones del Ministerio de la Administración y del Interior y en la Agencia Nacional de la Función Pública. Con el apoyo de la Red de Modernizadores, la implementación del MCE comenzó a extenderse a otros ministerios, distritos, prefecturas así como a otras instituciones interesadas.

Según la encuesta realizada a los modernizadores, la extensión de la implementación del MCE es muy lenta. Así, casi tres cuartas partes de los ministerios, prefecturas y consejos provinciales no han comenzado aún la implementación piloto.

GRÁFICO 3

Implementación del MCE en las instituciones públicas

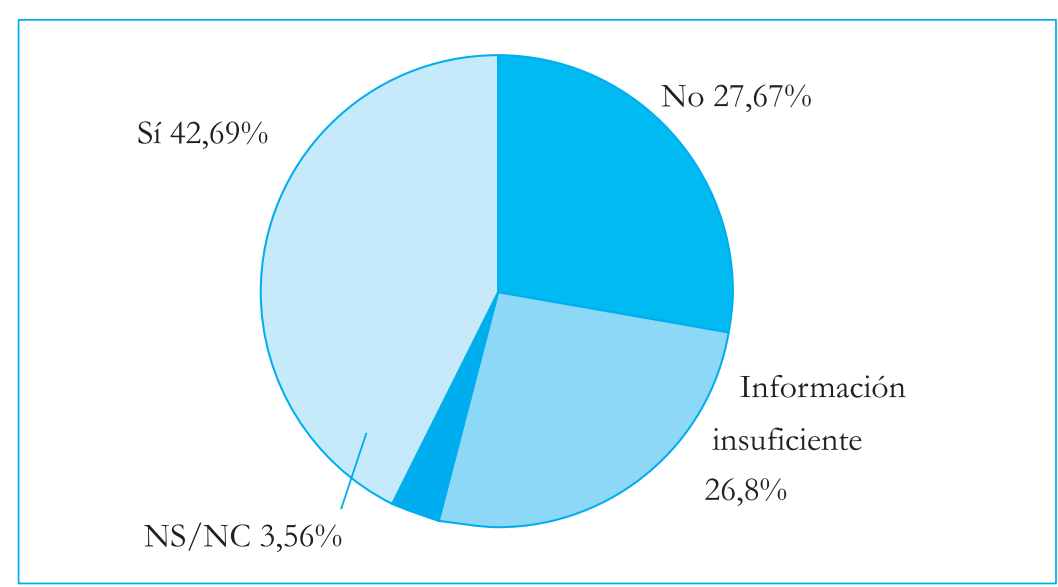




\section{Integración europea y Administración pública}

Los representantes de las comunidades locales creen que la integración en las estructuras de la UE es un proceso benéfico para el desarrollo local a corto y medio plazo. Más del $80 \%$ de los alcaldes respondieron que la integración en la UE influiría positivamente en el desarrollo de su comunidad en los próximos cinco años, mientras que un $17,8 \%$ eran escépticos.

TABLA 4

¿Qué influencia piensa que tendrá la adbesión de Rumanía en la UE en el desarrollo de su comunidad en los próximos 5 años?

\begin{tabular}{|l|c|c|}
\hline \multicolumn{1}{|c|}{ Opciones de respuesta } & Frecuencia relativa (\%) & $\begin{array}{c}\text { Frecuencia relativa } \\
\text { acumulada (\%) }\end{array}$ \\
\hline Influencia negativa & 2.4 & 2.4 \\
\hline Influencia insignificante & 15.4 & 17.8 \\
\hline Influencia positiva & 81.8 & 99.6 \\
\hline NS/NC & 0.4 & 100.0 \\
\hline Total & 100.0 & - \\
\hline
\end{tabular}

Desgraciadamente, el optimismo de los alcaldes sobre el proceso de adhesión no se ve acompañado por la necesaria capacidad de las autoridades y compañías locales para usar los fondos estructurales. Más aún, las administraciones locales no están interesadas en valorar la formación necesaria para acceder y emplear estos fondos de los funcionarios locales electos y de las compañías (el 15\% de los alcaldes entrevistados no pudo dar su opinión a este respecto).

\section{GRÁFICO 4}

\section{Formación de las compañías locales en el uso de los fondos estructurales}

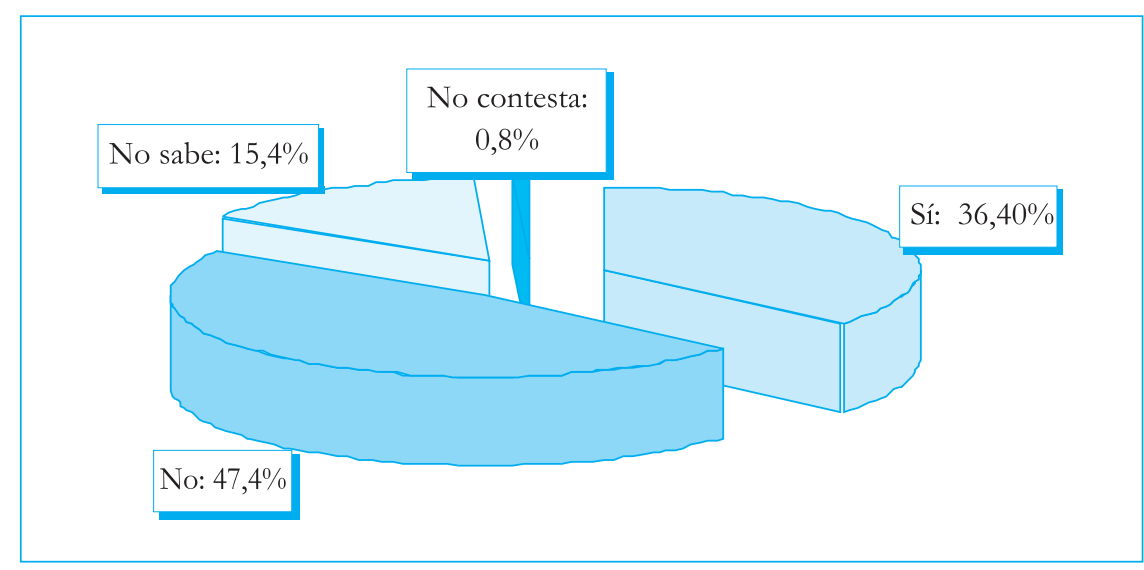

Más de la mitad de las autoridades locales en Rumanía aún no han solicitado fondos de la UE para el desarrollo institucional o el desarrollo de las comunidades locales. Esto es una clara indicación de la reducida capacidad administrativa para emplear los fondos estructurales que existirá en el período inmediatamente posterior a la adhesión. 
TABLA 5

Uso de financiación externa

\begin{tabular}{|l|c|}
\hline \multicolumn{1}{|c|}{ Opciones de respuesta } & Frecuencia relativa (\%) \\
\hline Ha usado fondos de la UE & 45.8 \\
\hline No ha usado fondos de la UE & 51.0 \\
\hline NS/NC & 3.2 \\
\hline Total & 100.0 \\
\hline
\end{tabular}

\section{Reforma de la Administración Pública Local}

\subsection{Definición del concepto de descentralización}

Descentralización es la transferencia de autoridad y responsabilidad sobre ciertas funciones públicas desde el nivel del gobierno central de un país a niveles subnacionales de gobierno u organismos autónomos (Rondinelli, Nellis y Cheema 1983).

La estrategia para la Reforma de la Administración Pública en Rumanía, aceptada por la Comisión Europea, define la descentralización a través de tres direcciones:

- Continuación de la descentralización, a través de la transferencia de responsabilidades administrativas y financieras y competencias desde la administración central a las autoridades locales;

- Continuación del proceso de desconcentración, a través de la delegación de responsabilidades a los niveles territoriales, de acuerdo a las necesidades locales, dentro de la misma estructura administrativa (los servicios desconcentrados operan bajo la autoridad del ministerio que delega esa responsabilidad);

- Transformación de los servicios territoriales desconcentrados, de acuerdo a las necesidades de los ciudadanos y para hacerlos más eficaces, en servicios descentralizados bajo el mando de las autoridades locales.

Dependiendo de las responsabilidades transferidas, la descentralización se corresponde a una de tres categorías: política, administrativa y fiscal.

Descentralización política significa un mayor poder para los ciudadanos en el proceso de toma de decisiones, garantizado por los procesos democráticos. Un argumento que apoya la descentralización es: «Las decisiones tomadas con una mayor participación de los ciudadanos están mejor fundamentadas y son más relevantes, incluyen un mayor interés social que aquellas decisiones tomadas al nivel de las autoridades políticas nacionales. Este concepto implica que los votantes logran conocer a sus representantes políticos mejor y, al mismo tiempo, los funcionarios electos conocen mejor las necesidades y expectativas de sus votantes» (Rondinelli 1999).

Este aspecto también se desprende de las respuestas dadas por los individuos entrevistados en la actual investigación. Así, aproximadamente el 50\% de las respuestas mostraron que hay una influencia política crónica en la Administración pública local. 
La descentralización administrativa es, según la literatura, la «transferencia de responsabilidad en la planificación, financiación y gestión de ciertas funciones públicas desde el gobierno central y sus agencias a unidades subordinadas, autoridades públicas semiautónomas o autoridades locales» (Rondinelli 1999).

Las subcategorías de la descentralización administrativa se definen frecuentemente de acuerdo al tipo de organismo o agencia que recibe la transferencia de responsabilidad.

En el actual contexto de la RAP, descentralización y desconcentración, como parte de la descentralización administrativa, son consideradas una prioridad, señalando que la descentralización se asimila a la devolución y a la delegación. En el mismo contexto, debemos señalar que, en el caso rumano, la devolución no implica una posible autonomía territorial y administrativa.

De acuerdo con la Constitución de Rumanía, enmendada en el 2003, «la Administración pública de las unidades administrativas y territoriales está basada en principios de descentralización, autonomía local y desconcentración de los servicios públicos». Al mismo tiempo, la ley fundamental establece que los Consejos Provinciales son «la autoridad de la Administración pública que coordina la actividad de los consejos locales, con vistas a proveer los servicios públicos de interés para la provincia» (Art. 122/1).

Durante los últimos 10 años, Rumanía ha realizado importantes progresos en el área de la descentralización. El proceso ha atravesado cuatro fases. En la primera fase (1991-1994) ${ }^{1}$ se realizaron cambios importantes en la estructura y financiación de las autoridades locales, incluyendo la introducción del sistema de impuestos locales. En la segunda fase de la política de reforma (1998-2000) la descentralización administrativa y financiera se convirtió en una prioridad. Basada en la nueva legislación sobre financiación de las autoridades públicas locales ${ }^{2}$, la parte del PIB destinado a los presupuestos locales se incrementó (de 3,6\% en 1998 a 6,5\% en 2000), pero también la parte del gasto local en el total del gasto público se incrementa (de 14,4\% en 1998 a 26,6\% en 2001). En la tercera fase (20012004) nuevas leyes establecen nuevas reglas para ciertas funciones de las autoridades locales ${ }^{3}$, específicamente para los servicios públicos ${ }^{4}$.

La cuarta fase (desde el 2004) comenzó con el diseño y aprobación de la Actualización de la Estrategia para la Aceleración de la Reforma de la Administración Pública (Decisión Gubernamental núm. 699/2004). Uno de los componentes más importantes de esta estrategia es la continuación de los procesos de descentralización y desconcentración. Para implementar esta estrategia, se esbozó un paquete legislativo en apoyo todo el proceso (la Ley Marco para la Descentralización núm. 339/2004, la Ley de Prefectos núm. 340/2004 y la Decisión Gubernamental núm. 2201/2004 sobre el Comité Técnico Interministerial y los Grupos de Trabajo sobre la Descentralización).

${ }^{1}$ Ley sobre Administración Pública Local núm. 69/1991, Orden Gubernamental núm.15/1992 sobre Impuestos Locales, y Ley núm.27/1994 sobre Impuestos Locales (República de Rumanía).

${ }^{2}$ La Ley núm. 6971991 y la Ley núm. 189/1998 sobre finanzas públicas locales fueron enmendadas (República de Rumanía).

${ }^{3}$ Ley núm. 215/2001 sobre Administración pública local (República de Rumanía).

${ }^{4}$ Ejemplos: Ley núm. 326/2001 sobre servicios públicos de la comunidad, Orden Gubernamental(GO) núm. 86/2001 sobre los servicios públicos locales de trasporte de pasajeros, GO núm. 84/2001 sobre el servicio público para el censo, GO núm. 88/2001 sobre los servicios públicos para las situaciones de emergencia, GO núm. 202/2002 sobre la gestión integral del área costera, GO núm. 21/2002 sobre la gestión de las comunidades urbanas y rurales, GO núm. 32/2002 sobre los servicios públicos para la distribución de agua y alcantarillado, GO núm.71/2002 sobre el establecimiento de servicios públicos locales para la gestión de terrenos de públicos y privados de interés local (República de Rumanía). 


\section{GRÁFICO 5}

¿Existe algún servicio público en su comunidad que mejoraría su prestación si fuera descentralizado desde el nivel central al nivel local?

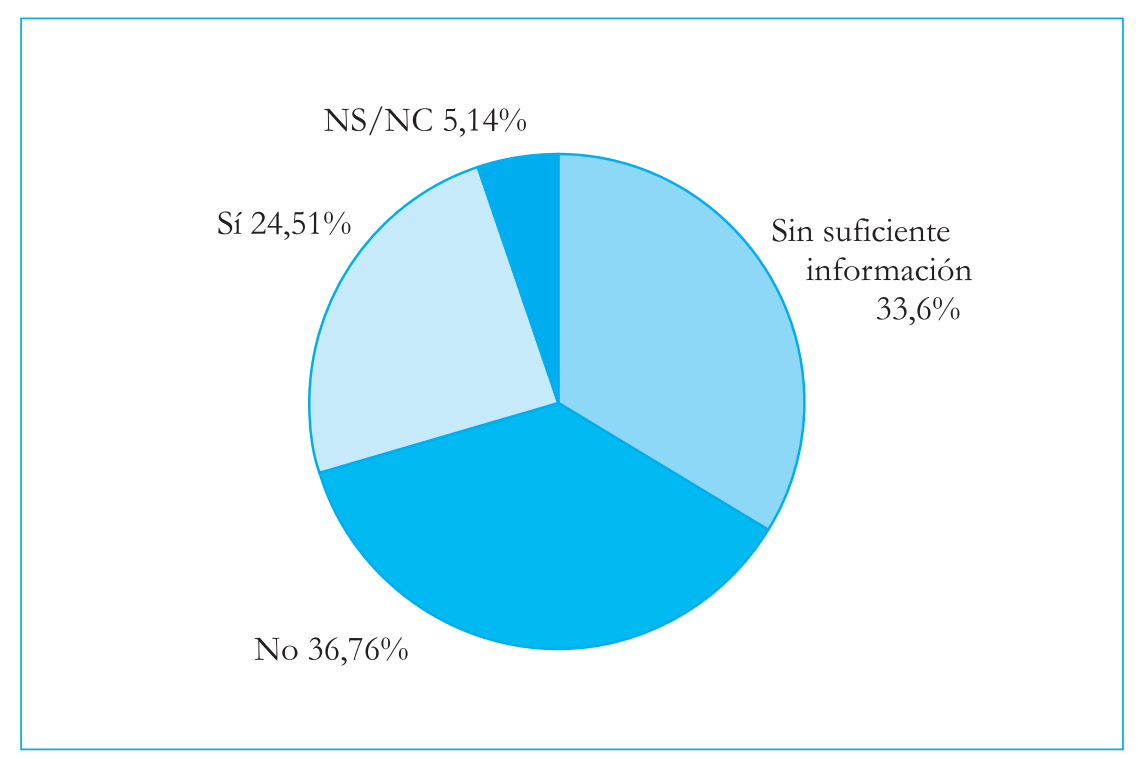

Las respuestas de los alcaldes nos permiten formular las siguientes conclusiones:

- Hay una opinión favorable hacia el proceso de descentralización y las ventajas que pueda generar;

- Las comunidades más pequeñas, que no se benefician de los servicios públicos proporcionados por el nivel central, tienen una opinión más negativa. De acuerdo a seguimientos realizados por expertos extranjeros, existe un fuerte sentimiento de frustración entre los funcionarios locales electos que piensan que la descentralización, definida por los textos legales está implementada insuficientemente y depende, en último término, de su habilidad para negociar con el Estado y aceptar el correspondiente riesgo político. Más aún, de acuerdo con estos expertos, la sensación de frustración es más fuerte en las comunidades más pobres que tienen menos recursos propios. Dado que las transferencias desde el gobierno central dependen esencialmente de los recursos provenientes del impuesto sobre la renta y el IVA recaudados en esa unidad territorial, se necesita un esfuerzo significativo de igualación para que las comunidades sean capaces de financiar las funciones básicas y obligatorias por ley. Es prácticamente imposible para estas comunidades generar los recursos para financiar las inversiones, aunque éstas sean necesarias. Por el contrario, las comunidades más ricas no tienen incentivos para movilizar su potencial fiscal y, a menudo, prefieren realizar deducciones o desgravaciones y solicitar al gobierno central ayuda para financiar el déficit.

- Un importante número de entrevistados no está familiarizados con el tema de la descentralización. 


\section{GRÁFICO 6 \\ ¿Existen algunos servicios públicos que debieran ser gestionados por el sector privado?}

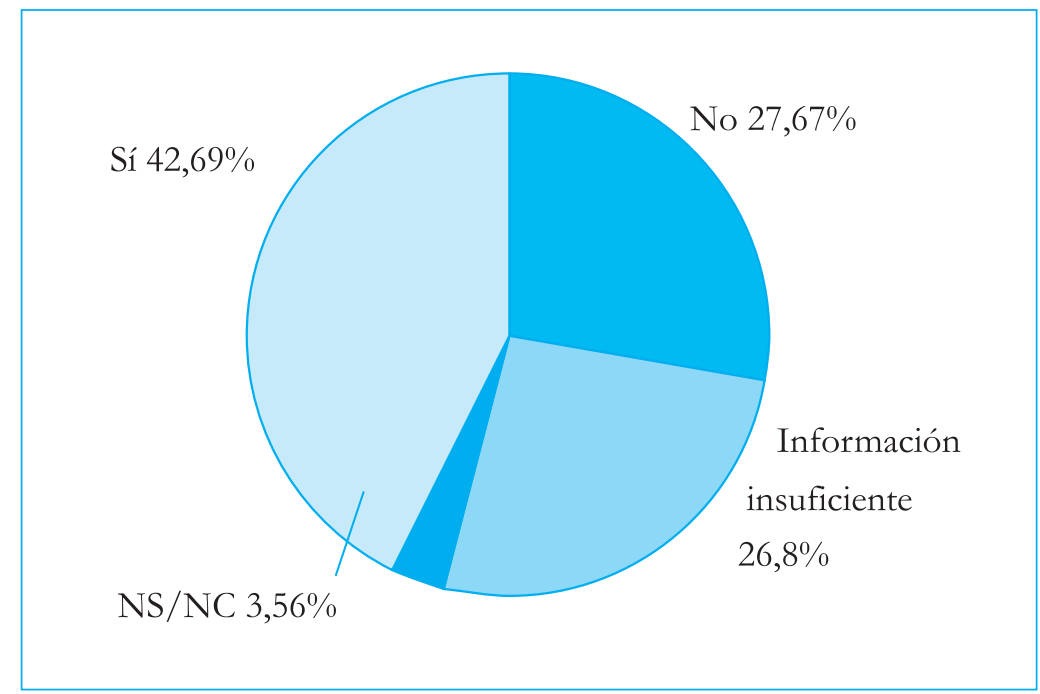

Los funcionarios locales electos entrevistados consideraron la posibilidad de privatizar algunos servicios públicos locales para hacerlos más eficaces y reducir los gastos del presupuesto local.

La descentralización fiscal se relaciona con un control mayor de las autoridades locales sobre los recursos financieros, tanto si se trata de distribuir gastos como de generar ingresos. Mucha de la literatura especializada en descentralización fiscal se centra en la naturaleza de las transferencias intergubernamentales y en las diferencias en la capacidad de generar ingresos entre varios tipos de organismos. Una característica básica de la descentralización fiscal es el equilibrio entre las responsabilidades y los recursos financieros que se necesitan para cumplir con las mismas.

Más del 60\% de los entrevistados cree que los pasos dados en la descentralización fiscal no son suficientes y, en algunos casos, son inadecuados para apoyar una apropiada financiación de los servicios públicos prestados a los ciudadanos.

Tanto los expertos rumanos como los extranjeros señalan que «los procesos de descentralización y desconcentración no han sido completados, lo que quiere decir que los recursos financieros necesarios no han sido transferidos y las competencias a transferir y los niveles de descentralización no han sido establecidos de manera precisa» (La Estrategia de la RAP, 2004).

La Unión Nacional de Consejos Provinciales y la Asociación de Directores Económicos de los Consejos Provinciales emprendieron una revisión detallada del actual estado de descentralización financiera en Rumanía y para las más importantes áreas de las políticas públicas (educación, salud, seguridad social, seguridad y orden público, agricultura, servicios de desarrollo local).

Junto a los aspectos positivos, se identificaron numerosas debilidades en el proceso de descentralización:

- Las administraciones públicas locales no tienen suficiente autoridad, lo que limita su capacidad de organizar los servicios eficientemente (e. g:: no tienen el derecho de fijar los precios de los servicios); 
- En algunas áreas pueden encontrarse aún mecanismos de control y decisiones discrecionales. Esto limita tanto la planificación como la previsión fiscal, y la posibilidad de introducir soluciones innovadoras a nivel local para proporcionar servicios más eficaces. De manera indirecta, esto limita la capacidad de absorción de los fondos de la UE;

\section{GRÁFICO 7}

\section{¿El actual sistema de financiación de los servicios públicos locales satisface sus necesidades?}

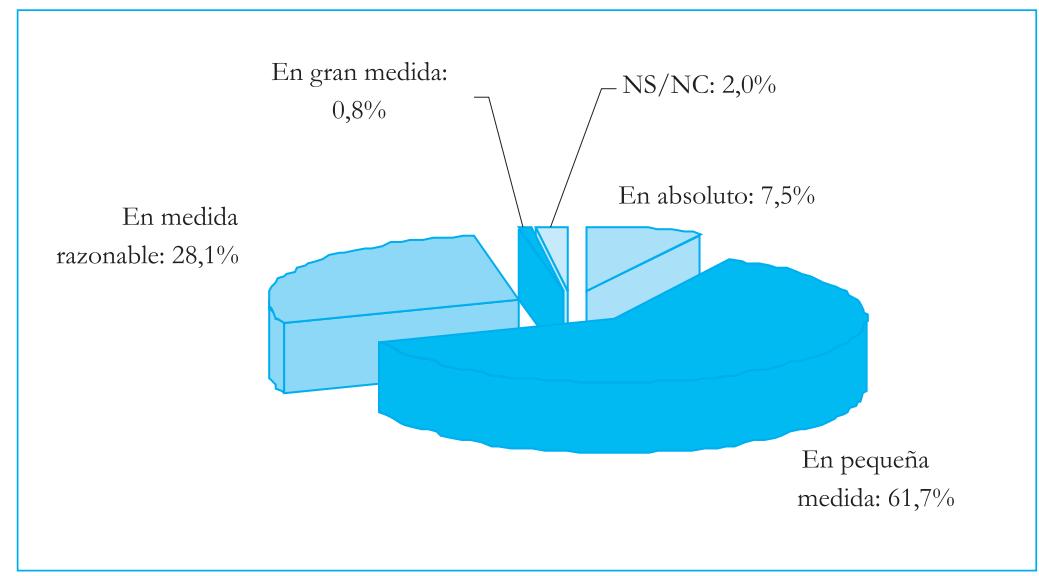

- La autonomía de la gestión financiera local está limitada por las regulaciones sobre la distribución de los propios ingresos, restringiendo el uso de las transferencias;

- El uso excesivo de distribuciones fijas está limitando el gasto efectivo de dinero, porque evita la coordinación y la integración de los servicios locales;

- Los mecanismos de igualación existentes no aseguran la equidad del sistema;

- La transferencia incompleta de propiedad es un límite a la gestión efectiva de los activos locales;

- Falta de garantías legales específicas y constitucionales para la autonomía local;

- Políticas públicas insuficientemente fundamentadas y parcialmente implementadas no pudieron dar soluciones racionales a los asuntos en cuestión.

- El excesivo uso de los procedimientos legislativos de urgencia, en lugar de los adecuados procesos de consulta;

- Las autoridades públicas no siempre tienen formación especializada en gestión financiera y gestión descentralizada de servicios;

- El desfase entre la responsabilidad para la toma de decisiones transferida a las administraciones locales y los recursos asignados para financiar estas decisiones (los recursos locales asignados no se corresponden con el aumento de las responsabilidades).

Un proceso de descentralización malamente gestionado puede producir una amplia variedad de fracasos estatales, desde «errores por omisión», cuando el Estado es incapaz de mejorar el rendimiento económico o administrativo, a «errores por acción», cuando las acciones del Estado conducen a un peor rendimiento económico (Krueger 1990: 923). Estos errores no sólo evitan el desarrollo de una descentralización política significativa destinada a mejorar la prestación de los servicios locales, sino que tienen además un impacto negativo sobre la credibilidad de las autoridades. 
El Regular Report de 2004 de la Comisión Europea establece: «Más importante aún: las autoridades rumanas han llevado a cabo esfuerzos considerables para desarrollar la estrategia que guía el proceso de descentralización de un modo estable y transparente. La estrategia se adoptó en Mayo de 2004 e identifica prioridades precisas para futuras reformas. Es de notar que la estrategia fue preparada tras un amplio debate público (un Forum Nacional) con los principales responsables. Pero las reformas propuestas aún están en fase de preparación y su implementación tiene que ser asegurada. La cooperación efectiva entre el Ministerio de la Administración y del Interior y el Ministerio de Finanzas Públicas necesita ser mejorada considerablemente» (p. 17).

\section{GRÁFICO 8}

¿Conoce algún servicio público que baya sido descentralizado en los últimos cuatro años?

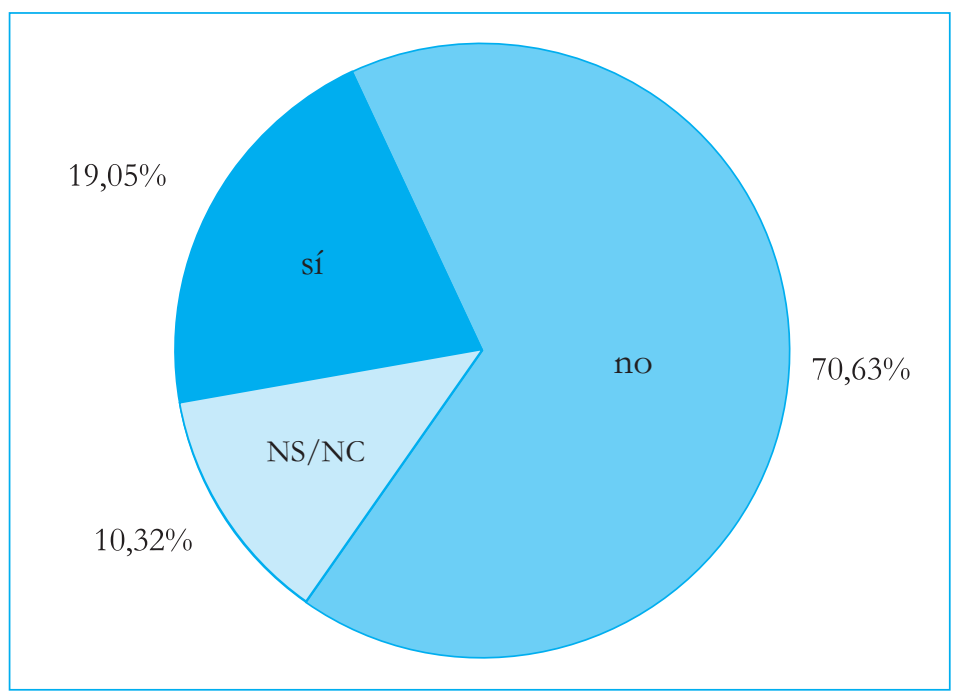

GRÁFICO 9

En su opinión y considerando el actual estado de descentralización, ¿̇en qué medida puede la Administración local cumplir las siguientes funciones?

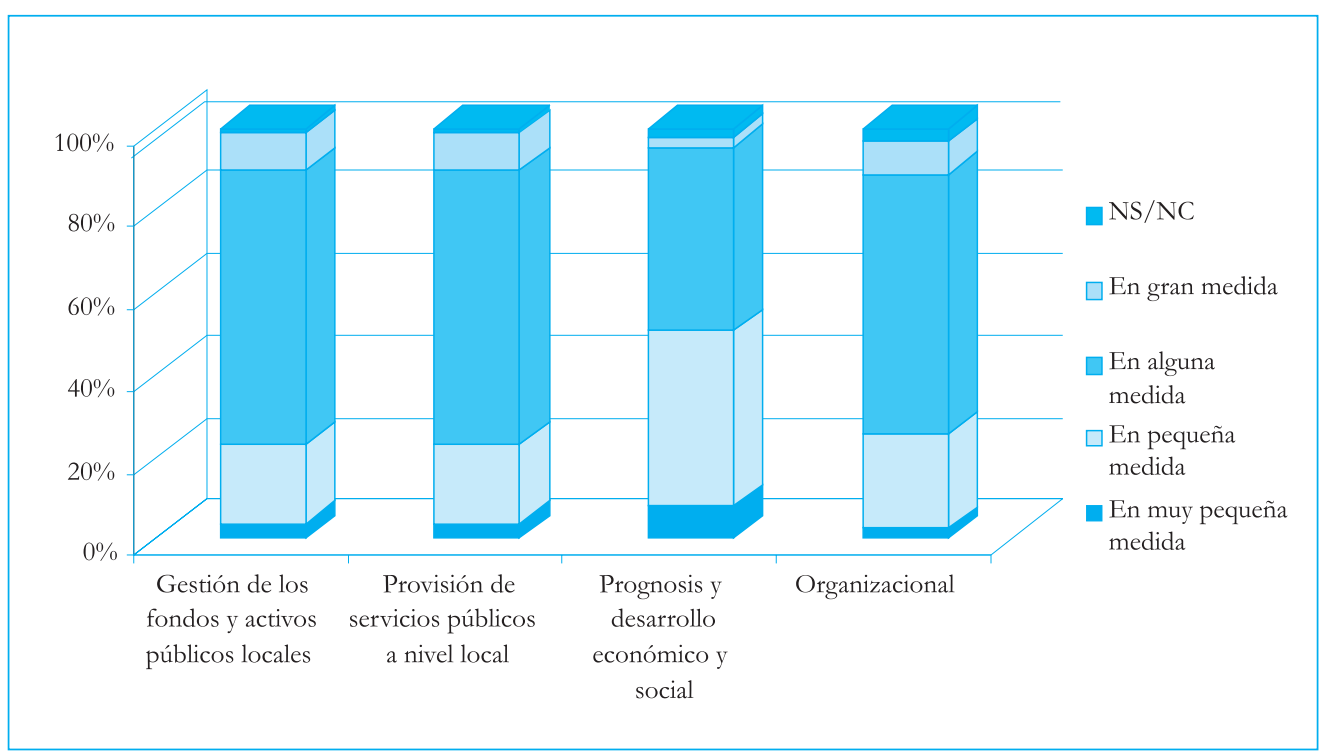


De las respuestas de los entrevistados podemos concluir que la descentralización no ha tenido efectos consistentes a nivel local. Posibles explicaciones de esto pueden ser:

Sólo el marco legislativo e institucional ha sido desarrollado y los resultados actuales a nivel local sólo serán evidentes en los próximos años;

- El proceso no era coherente, ni estaba adecuadamente analizado, los recursos necesarios no fueron proporcionados y la comunicación con los responsables ha sido deficiente;

- No todas las unidades administrativas territoriales se han visto envueltas en el proceso, dada su insuficiente capacidad administrativa y de gestión. Esto también se recoge en el Regular Report de la Comisión Europea de 2004: «La mayoría de las autoridades locales sufre de una limitada capacidad administrativa y los funcionarios cambian con excesiva frecuencia» ${ }^{11}$.

La respuesta de los alcaldes revela, entre otras causas, una débil implementación de las leyes de administración local y falta de transparencia del proceso de descentralización

\section{GRÁFICO 10}

Durante su permanencia en el puesto, ¿alguna vez bizo propuestas a favor del proceso de descentralización?

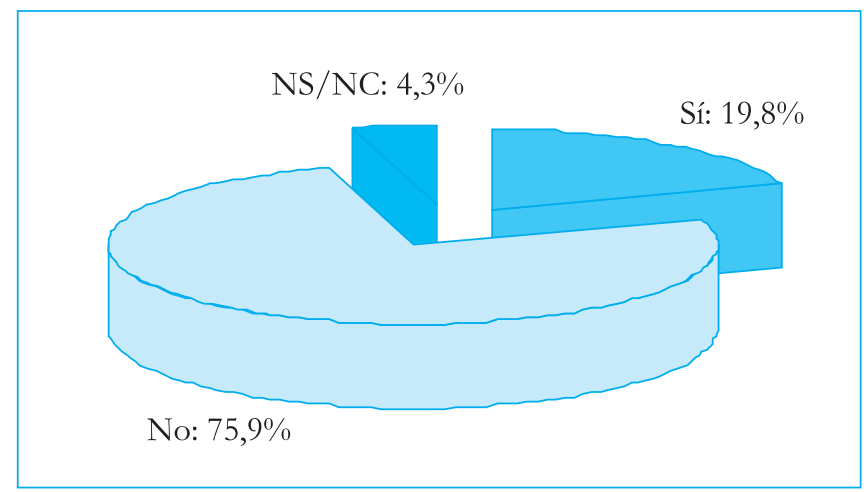

\section{Riesgos del proceso de reforma}

Un aspecto importante de la RAP es la gestión del proceso de implementación de la reforma. El mayor riesgo es la sobrecarga con tareas rutinarias, sin dejar tiempo para el diseño de nuevas soluciones a los problemas encontrados.

Para que la RAP sea exitosa es necesario que un gran número de grupos objetivo (especialmente la gente clave en los niveles de gestión y toma de decisiones) apoyen y encomienden la necesidad de cambios y su implementación. Basado en el modelo de desarrollo organizacional integrado, las influencias negativas en el proceso de implementación pueden ser identificadas: factores estratégicos (complejidad del proceso de toma de decisiones), factores estructurales (burocracia del sistema, recursos humanos y financieros limitados, tamaño y complejidad), factores culturales (aversión al riesgo, inercia, mentalidad), y factores actitudinales (falta de incentivos individuales, incomprensión de los objetivos generales, frustración, actitud expectante). 
La Administración pública no puede ser reformada en unos pocos años. Es un proceso a largo plazo, que probablemente sólo puede ser implementado por numerosos gobiernos consecutivos en un entorno difícil, altamente competitivo y sometido a rápidos cambios. Por esta razón, es necesario alcanzar un acuerdo sobre los siguientes principios que gobernarán la totalidad del proceso de reforma:

- Amplia diseminación de la información sobre la reforma y movilización del interés de los ciudadanos, los profesionales, los representantes políticos y los funcionarios públicos en que se produzca la reforma y en el intercambio democrático de opiniones sobre lo deseable y factible de su evolución.

- Basar la reforma en sólidos análisis profesionales del estado actual y la prestación de la Administración pública y en periódicas evaluaciones de las consecuencias de los pasos ya dados.

- Usar la experiencia de la reforma de la Administración pública en otros países, particularmente en los Estados miembros de la UE y en los países que se están preparando para la adhesión a la misma, al tiempo que se consideran nuestras propias tradiciones y experiencias.

- Adoptar una aproximación comprehensiva hacia la reforma: los cambios parciales o aislados no deberían implementarse si no se conciben como partes integrantes y orgánicas del proceso y la estrategia de reforma.

- Ver la reforma como un proceso abierto: los componentes individuales de la reforma serán continuamente actualizados y adaptados a los cambios en el entorno de la Administración pública y en los otros componentes de la reforma, y utilizarán la experiencia adquirida durante la implementación.

- Determinar prioridades estratégicas: un número limitado de cambios prioritarios tendrán que ser definidos para cada fase de la reforma en los que la atención y los fondos habrán de ser concentrados; éstos deberían ser los cambios que predeterminen la totalidad del progreso de reforma y condicionen o influencien al resto de cambios.

- Asegurar la continuidad de la operatividad de la Administración pública, que debe continuar funcionando también durante el proceso de reorganización, descentralización y otros cambios.

- Gestionar y coordinar la totalidad de la reforma desde un único centro al mayor nivel del gobierno, para asegurar la coherencia de la implementación de la estrategia.

\section{Corrupción en la Administración pública}

En los últimos años, el ambiente académico e investigador y las organizaciones internacionales (fundamentalmente el Fondo Monetario Internacional y el Banco Mundial) han mostrado un creciente interés en medir el nivel de corrupción y de economía sumergida en cada país. Alguno de los más importantes trabajos sobre medición de la corrupción y su impacto sobre el desarrollo económico incluyen: A. Krueger (1974), S. RoseAckerman (1975), P. Mauro (1995), P. Bardhan (1997), V. Tanzi (1998), Shang-Jin Wei (2001), etc. Por sus efectos, los dos fenómenos determinan una significativa reducción de los recursos financieros disponibles para cada Estado, así como un descenso en la eficiencia en el uso de estos fondos.

Para evaluar el nivel de corrupción de un país han sido establecidos una serie de indicadores (Wei 2001), principalmente: International Country Risk Index, de la International Country Risk Guide (ICRG), basado en «opiniones de expertos»; el índice de corrupción del Global Competitiveness Report (GCR) y el World Development Report (WDR), basado en encuestas a ciudadanos y compañías; el índice de corrupción percibida, de Transparency 
International (TI), basado en un «indicador de indicadores»; el indicador de corrupción basado en numerosos datos objetivos y relevantes.

El cálculo del índice de corrupción en un país está basado en las respuestas de los hombres de negocios extranjeros que están en dicho país. Un indicador significativo usado para medir el nivel de corrupción es el Transparency International Corruption Index (TICI) (Graf 1999). Entre este índice y el Human Development Index (HDI) hay una fuerte conexión. Para los países desarrollados, un nivel alto en el HDI implica un TICI bajo, mientras que en los países subdesarrollados un bajo HDI manifiesta una corrupción endémica en la sociedad. Por ejemplo, para las series de valores de TICI y HDI recogidas en el mundo en 2001, el coeficiente de correlación linear es 0,77 . Para los miembros de la OTAN el valor es 0,71 .

Antes de 1990, la economía sumergida y la corrupción existían en los países de Europa Central y del Este bajo varias formas. Más tarde, los procesos de transición se vieron acompañados de un incremento de corrupción y la consolidación de las economías sumergidas. En 2001 en Rumanía, casi el 30\% de la población vive en la pobreza. Para el mismo año, el índice de corrupción sitúa a Rumanía entre los países del Este más corruptos.

En conclusión, «si la economía sumergida en los países más pobres permitía la creación de cierto número de puestos de trabajo en el deseo de lograr solidaridad y cohesión familiar, su excesivo desarrollo es un verdadero peligro tanto para la economía como para el sistema político de ese país» (Germanangue - Debare 1996: 22-24).

De acuerdo con la Comisión Europea, «en las áreas de descentralización y administración local, los temores expresados en el Regular Report del último año son aún válidos. La transferencia de competencias a las autoridades locales no fue acompañada de la correspondiente transferencia de recursos.

La capacidad para incrementar los ingresos locales permanece limitada, la legislación sobre transferencias a las autoridades locales no es transparente, concediendo a los consejos provinciales un importante control funcional sobre los gastos de los consejos locales. Los fondos adicionales para la inversión local (particularmente carreteras y sistemas de calefacción) se proveen a través de «fondos especiales» que provienen del gobierno central. Los organismos responsables de la gestión de los fondos públicos no tienen poder a nivel local, y sus informes ocultan el uso de fondos públicos en beneficio de ciertos grupos de interés. Se trata de un asunto de importancia en un momento en el que el país se está preparando para la gestión de los fondos estructurales de la UE.

De las respuestas de los entrevistados, tanto de aquellos que ocupan puestos de toma de decisiones (alcaldes), como de aquéllos encargados de la implementación (modernizadores) podemos identificar la corrupción como una influencia negativa importante en las actividades habituales, pero también en el proceso de reforma mismo.

Los siguientes factores fueron considerados como causas para este fenómeno:

- El marco legal: éste permite en gran medida la corrupción en la Administración pública local. Nuestra recomendación fue acelerar el proceso de revisión de la actual legislación que regula las actividades de la Administración pública local, de acuerdo a los requisitos de la UE y el Banco Mundial sobre la RAP.

- El sistema retributivo: es el principal factor que mantiene la corrupción en el sistema, de acuerdo con las respuestas. Eso resulta evidente, considerando que el nivel de los salarios no se corresponde con las responsabilidades en el sector público. Igualmente, está determinado por la falta de una estrategia sobre la remuneración del funcionariado y el desarrollo de un sistema unificado de retribuciones que siga esa 
estrategia. Esto sería un instrumento capaz de crear el tipo adecuado de incentivo para los funcionarios, atrayendo y manteniendo a los mejores en la administración y cubriendo los vacíos entre las diferentes categorías de funcionarios, y también entre los sectores público y privado.

\section{GRÁFICO 12}

¿Piensa que la corrupción es un problema genuino de la Administración pública?

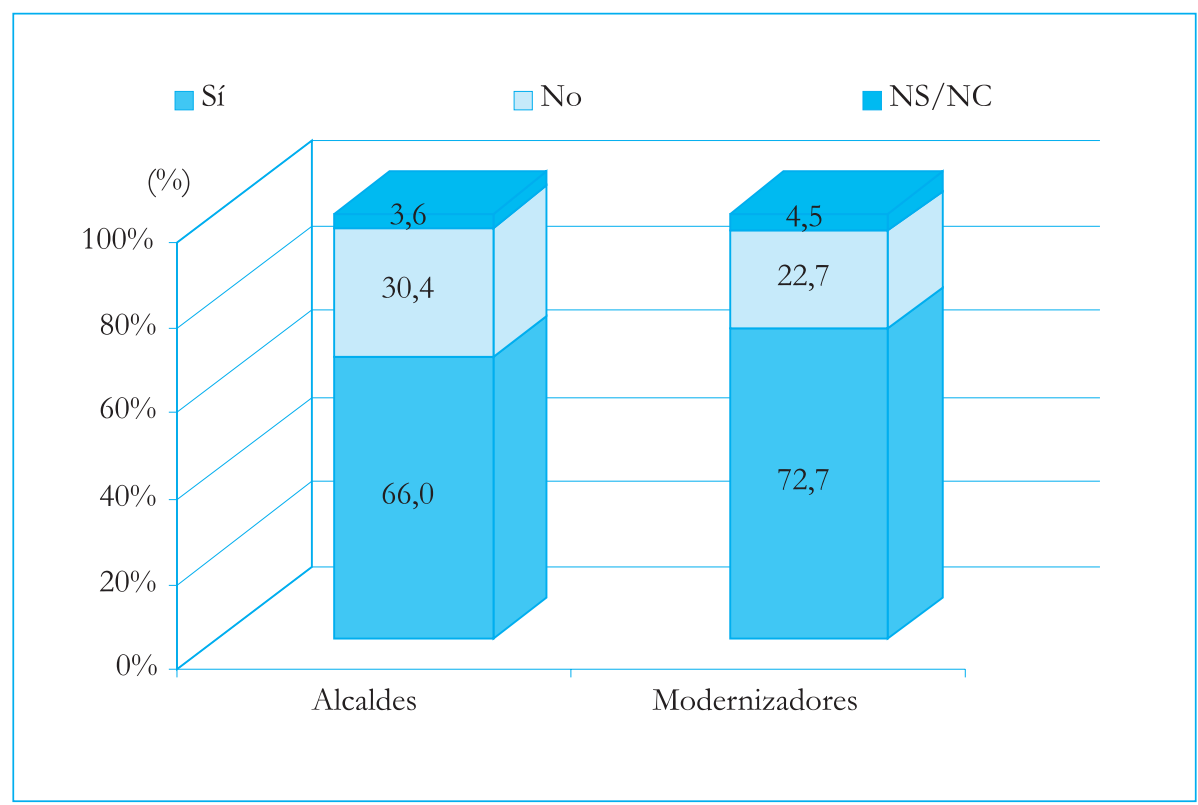

- Ética de los funcionarios: es el último factor citado en la encuesta, pero no es insignificante. La explicación para ello es la escasa conciencia de la cultura organizacional, la mentalidad trasnochada de los funcionarios que contradice las tendencias fundamentales de la sociedad. Es importante señalar que los modernizadores mencionaron este factor mucho más a menudo que los alcaldes. Ello puede explicarse porque están más próximos a los ciudadanos que los encargados de tomar decisiones.

- Presiones de los intereses económicos: es un factor importante en la generación de la corrupción, y es sentido con mayor fuerza por los alcaldes que por el personal técnico, porque los encargados de tomar decisiones son objetivos más adecuados para este tipo de presión.

- Presión del sistema político: también es un factor importante. Casi el 50\% de los entrevistados dijeron que la influencia política es amplia o muy amplia y favorece la corrupción. Las explicaciones pueden ser: el alto nivel de inestabilidad de los funcionarios, especialmente en los niveles de gestión, con los ciclos electorales y el «nepotismo» político como preocupaciones fundamentales en la distribución de los recursos financieros a nivel local. 
TABLA 6

Factores que fomentan la corrupción - Alcaldes

\begin{tabular}{|l|r|r|r|c|c|c|}
\hline \multicolumn{1}{|c|}{ Opciones de respuesta } & Marco legal & $\begin{array}{c}\text { Sistema } \\
\text { salarial }\end{array}$ & $\begin{array}{c}\text { Ética del sector } \\
\text { público }\end{array}$ & $\begin{array}{c}\text { Presiones de } \\
\text { intereses } \\
\text { económicos }\end{array}$ & $\begin{array}{c}\text { Presión del } \\
\text { sistema político }\end{array}$ & $\begin{array}{c}\text { Comportamiento } \\
\text { ciudadano }\end{array}$ \\
\hline En absoluto & 3.6 & 0.0 & 3.0 & 6,0 & 13.2 & 6.6 \\
\hline De forma insignificante & 12.6 & 0.6 & 19.9 & 18,0 & 12.6 & 24.1 \\
\hline Moderadamente & 15.6 & 5.4 & 32.5 & 19,2 & 15.0 & 28.9 \\
\hline Bastante & 29.9 & 25.3 & 19.9 & 30,5 & 27.5 & 19.3 \\
\hline En gran medida & 23.4 & 65.7 & 17.5 & 12,0 & 21.6 & 7.8 \\
\hline NS/NC & 15.0 & 3.0 & 7.2 & 13,8 & 10.2 & 13.3 \\
\hline Total & 100.0 & 100.0 & 100.0 & 100,0 & 100.0 & 100.0 \\
\hline
\end{tabular}

\section{GRÁFICO 14}

Factores que fomentan la corrupción - Alcaldes

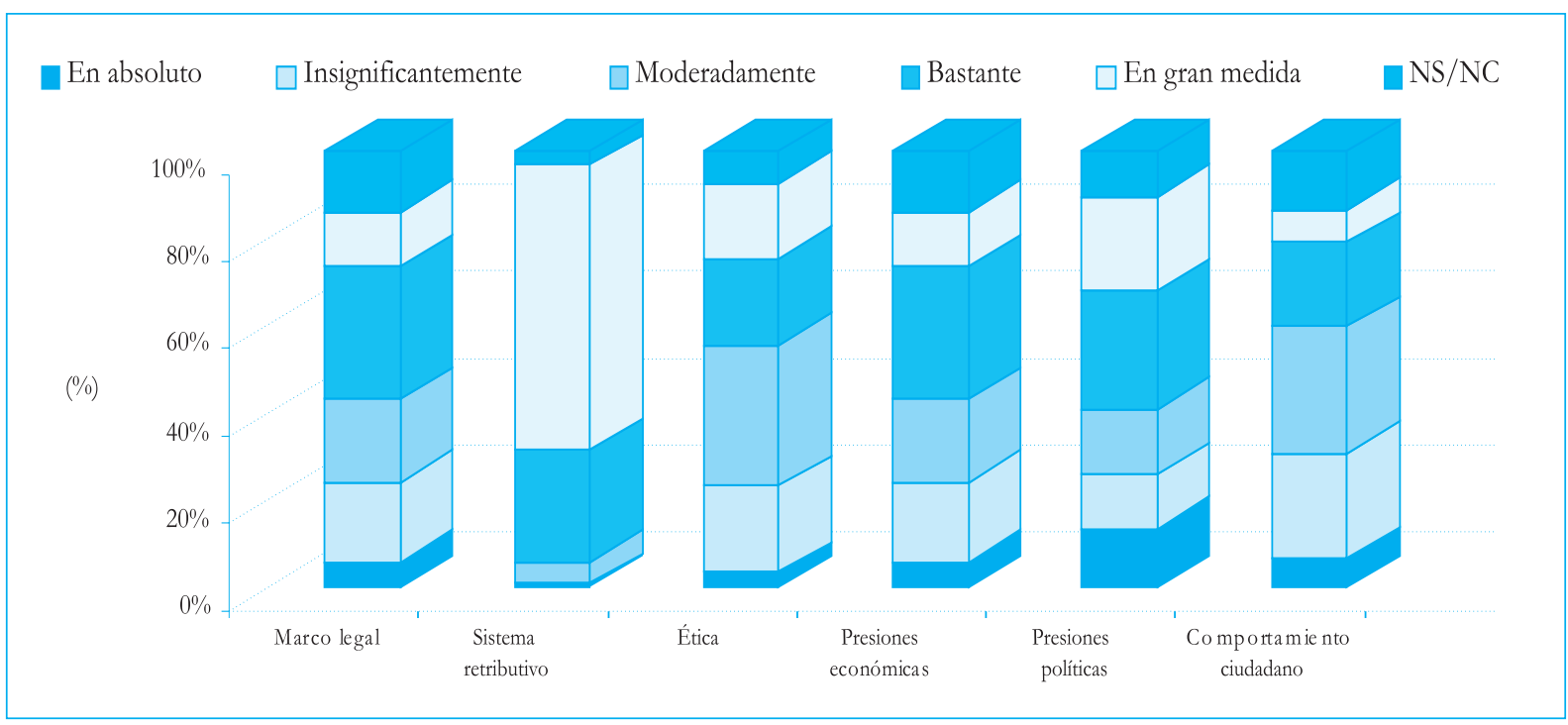

TABLA 7

Factores que fomentan la corrupción - Modernizadores

\begin{tabular}{|l|r|r|r|r|r|r|}
\hline \multicolumn{1}{|c|}{ Opciones de respuesta } & Marco legal & $\begin{array}{c}\text { Sistema } \\
\text { salarial }\end{array}$ & $\begin{array}{c}\text { Ética del sector } \\
\text { público }\end{array}$ & $\begin{array}{c}\text { Presiones de } \\
\text { intereses } \\
\text { económicos }\end{array}$ & $\begin{array}{c}\text { Presión del } \\
\text { sistema político }\end{array}$ & $\begin{array}{c}\text { Comportamiento } \\
\text { ciudadano }\end{array}$ \\
\hline En absoluto & 0.0 & 0.0 & 0.0 & 0.0 & 12.5 & 0.0 \\
\hline De forma insignificante & 6.3 & 6.3 & 25.0 & 12.5 & 12.5 & 43.8 \\
\hline Moderadamente & 18.8 & 12.5 & 18.8 & 31.3 & 25.0 & 12.5 \\
\hline Bastante & 50.0 & 31.3 & 50.0 & 25.0 & 18.8 & 18.8 \\
\hline En gran medida & 12.5 & 50.0 & 6.3 & 18.8 & 25.0 & 25.0 \\
\hline NS/NC & 0.0 & 0.0 & 0.0 & 12.5 & 6.3 & 0.0 \\
\hline Total & 100.0 & 100.0 & 100.0 & 100,0 & 100.0 & 100.0 \\
\hline
\end{tabular}




\section{REFERENCIAS BIBLIOGRÁFICAS}

Alesina, A. y Perotti, R. (1993), «The Politics of Economic Growth», Working Paper 4341. Cambridge, Mass.: Harvard University. Alesina, A. y Perotti, R. (1993), «Income Distribution. Political Instability and Investment», Working Paper 4486, NBER. Cambridge, Mass.

Andrei, T. (2002), «The Costs of Security of Aspiring Countries - Quantitative Models», Working Paper. Bruselas: OTAN.

Azam, J.P. (1995), «How to pay for the peace? A theoretical Framework with References to African Countries», Public Choice 83: 173-184.

Azam, J.P. (1995), «Risque politique et croissance en Afrique». Comunicación en el Congreso de la AFSE. París.

Balls, A. (1999), «Bombing costs present allies with hefty bill», Financial Times, 9 de abril.

Banco Mundial (1993), World Development Report 1993. Washington DC: Oxford University Press.

Bardhan, P. (1997), «Corruption and development: a review of issues», Journal of Economic Literature 35.

Barro, R. (1991), «Economic growth in a cross-section of countries», Quarterly Journal of Economics 106: 407-443.

Besnainou, D. (1995), «Les fonds structurels: quelle application aux PECO? Transition et élargissement», Economie Internationale 62.

Buffotot, P. (1989), «La défense en Europe-Les adaptations de l'après-guerre froide», Notes et Etudes documentaires 5064-65. París.

Chevalier, A., Lemoine, F. y Nayman, L. (1999), «L’Union Européenne et sa périphérie. Conséquences de l'intégration commerciale de l'Europe centrale», Revue Economique 6.

Comisión Europea (2004), Regular Report on Romania's progress towards EU accession. Bruselas: UE.

Fischer, S., Sahay, R. y Vegh C.A. (1998), «How far is Eastern Europe from Brussels?», Working Paper 98/53. FMI.

Germanangue-Debare M. (1996), «Les pièges de l'économie souterraine», Le Courrier de l'UNESCO, junio: 22-24.

Gobierno de Rumanía, Law on Local Public Administration no. 69/1991, Government Ordinance no.15/1992 on Local Taxes, and Law no.27/1994 on Local Taxes. Bucarest.

Graf L.M J. (1999), «Transparency International Corruption Index, Responding to the Challenges of Corruption». Actas de la Conferencia Internacional sobre Corrupción: 257-277. Milán.

Gupta S., De Mello, L. y Sharan R. (2001), «Corruption and military spending», European Journal of Political Economy 17: 749-777.

Gupta, D. (1991), The Economics of Political Violence. Nueva York: Praeger.

Mauro, P. (1995), «Corruption and growth», Quarterly Journal of Economics 110.

Mauro, P. (1996), The Effects of corruption on Growth, Investment, and Government Expenditure. Washington: FMI.

Mauro, P. (1998), «La corruption: causes, conséquences et recommandations», Finance et Développement, 35.

Hutchinson, P. L. y LaFond, A. K. (2004), Monitoring and Evaluation of Decentralization Reforms in Developing Country Health Sectors. Tulane: Tulane University.

PNUD, (1990-2001), Human Development Report. Publicado por el. Nueva York: Oxford University Press.

Profiroiu, M. (2003), Managementtul Strategic al Colectivitatilor Locale. Bucarest: Editura Economica.

Profiroiu, M. y Andrei, T. (2005), «Reforma administratiei publice in contextual integrarii europene», Working Paper. Bucarest: Instituto Europeo de Rumanía.

Rondinelli, D.A., Nellis, J. R. y Cheema, G. S. (1983), «Decentralization in Developing Countries: A. Review of Recent Experience». Staff Working Papers 581. Washington DC: Banco Mundial. 
Rondinelli, D.A. (1999), «What is Decentralization?», en J. Litvack y J. Seddon, eds., Decentralizatin Briefing Notes, Washington DC: Instituto del Banco Mundial.

Rose-Ackerman, R. (1975), «The economics of corruption», Jorunal of Public Economics 5617.

Tanzi, V. (1998), Corruption Around the World: Causes, Consequences, Scope and Cures. Washington DC: FMI.

Wei Shang-Jie. (2001), «Corruption in Economic Development: Grease or Sand?», Economic Survey of Europe 2. ONU.

Yost D., (1988), NATO Transformed. The Alliance's New Roles in International Security. Washington DC: United States Insitute of Peace Press. 